Images du travail, travail des images

$8 \mid 2020$

Filmer, travailler, chercher

\title{
Des films pour analyser le travail et documenter des situations de formation
}

Producing films about work to analyse work practices and document training situations

Marc Durand, Annie Goudeaux, Germain Poizat et Juana

Sarmiento Jaramillo

\section{OpenEdition}

\section{Journals}

Édition électronique

URL : http://journals.openedition.org/itti/306

DOI : $10.4000 /$ itti.306

Éditeur

Université de Poitiers

Référence électronique

Marc Durand, Annie Goudeaux, Germain Poizat et Juana Sarmiento Jaramillo, « Des films pour analyser le travail et documenter des situations de formation », Images du travail, travail des images [En ligne], 8 | 2020, mis en ligne le 01 février 2020, consulté le 14 avril 2021. URL : http:// journals.openedition.org/itti/306 ; DOI : https://doi.org/10.4000/itti.306

Ce document a été généré automatiquement le 14 avril 2021.

Images du travail, travail des images 


\section{Des films pour analyser le travail et documenter des situations de formation}

Producing films about work to analyse work practices and document training situations

Marc Durand, Annie Goudeaux, Germain Poizat et Juana

Sarmiento Jaramillo

Les auteurs remercient Luc Ria, Jacques Saury et Philippe Veyrunes pour leur lecture critique d'une version antérieure de cet article.

1 Être filmé au travail, observer et commenter des films représentant ce travail, visionner et analyser ses propres prestations ou celles des autres, sont des pratiques qui font de longue date partie de l'univers de la formation des adultes. Ce recours au filmage du travail et l'utilisation des films à des fins d'apprentissage-développement soulève des questions appelant des réponses pratiques, mais surtout l'élaboration d'une ingénierie de formation consistante.

2 Nous décrivons dans cet article des éléments d'une telle ingénierie des situations de formation (Durand 2008; Durand et Poizat 2015), qui a pour caractéristique de s'articuler aux études sur l'activité humaine et ses transformations dans les situations quotidiennes (Poizat, Durand et Theureau 2016). Cette ingénierie a pour objectifs de définir précisément les contenus à acquérir, et de se protéger contre le risque de valoriser des acquisitions marginales par rapport à l'expertise professionnelle visée. $\mathrm{Ne}$ se satisfaisant ni d'une connaissance générale et globale du travail, ni de référentiels de métier prescriptifs, elle s'intéresse à l'activité en situation des professionnels et des apprentis.

3 Nous nous centrons ici sur le tournage et le montage de films sur le travail, en insistant sur le fait que l'un et l'autre sont contraints par la nature du travail en question, par les présupposés et exigences théoriques et méthodologiques de la démarche d'analyse du travail, et par les effets recherchés du recours à ces films en cohérence avec les 
hypothèses qui fondent la démarche formative. La présentation concerne deux aspects principaux : d'une part le concours de l'image filmée à l'analyse préalable du travail (principes d'observation et analyse de l'activité, modalités d'enregistrement des traces de l'activité de travail support de séquences d'auto-confrontation et de repérage de ses contraintes et effets extrinsèques); d'autre part la conception (choix des contenus et principes de montage) de films portant sur le travail en vue de documenter des situations de formation.

4 Cet article est illustré à partir d'une intervention pilote réalisée sous l'égide de l'université de Genève, en partenariat avec l'office des poursuites du canton de Genève, et le service de formation de l'état. Celle-ci a fait suite à l'implémentation problématique d'un nouveau logiciel et à la restructuration des services concomitante d'une mise en question de la gestion de l'office dans les médias locaux, le tout dans un contexte d'interrogation sur la politique cantonale de services publics. L'intervention avait pour objectifs d'analyser l'activité au travail des huissiers, de contribuer à la constitution d'un référentiel de leur métier afin d'alimenter des programmes de formation initiale et continue, et d'aider aux décisions managériales et de réorganisation dans les services.

\section{Le travail des huissiers}

\section{La fonction d'huissier et l'office des poursuites du canton de Genève}

5 La fonction d'huissier existe dans de nombreux pays avec des variantes. En France l'huissier de justice est un officier public et ministériel exerçant une profession libérale réglementée. Il a, seul, qualité pour exécuter les décisions de justice et délivrer des actes. Il exerce aussi sans en avoir le monopole, dans des domaines tels que le recouvrement amiable de créances, l'aide à la rédaction des actes sous seing privé, les consultations juridiques, l'administration d'immeubles, les ventes aux enchères publiques... En Suisse, une loi fédérale règle la procédure de recouvrement de créances, qui est menée par des offices administratifs cantonaux : les offices des poursuites. Les huissiers sont des fonctionnaires employés du canton et en poste dans ces offices cantonaux, ils n'ont pas de compétence judiciaire; ils exécutent la procédure de recouvrement sur réquisition du créancier.

6 Au sein de l'office des poursuites les huissiers accomplissent notamment des actes de prise de connaissance des réquisitions de poursuite par les créanciers, des continuations et des réalisations (exemples : lorsqu'un débiteur fait déjà l'objet d'une plainte ou a été considéré comme insolvable en raison de revenus insuffisants pour assurer un minimum vital, ou fait l'objet d'un prélèvement sur salaire...). Les huissiers réalisent aussi les notifications de commandements de payer, et l'encaissement des paiements effectués par les débiteurs. Ils procèdent également aux saisies, aux ventes des biens saisis et à la distribution de leurs produits aux créanciers. Enfin, ils délivrent des actes de défaut de biens lorsque le débiteur est jugé insolvable.

7 Les huissiers caractérisent leur métier comme imposant des arbitrages souvent délicats entre créanciers et débiteurs. Ils pointent un contraste structurant entre le caractère massif et en croissance du phénomène social d'insolvabilité, détresse sociale et délinquance d'une part, et les moyens juridiquement et pratiquement limités de leur action d'autre part. Ils insistent sur le fait que leur travail est usant et nécessite des 
efforts pour tenir. Pour cela ils développent des stratégies d'auto-protection et de détachement: ils se blindent, contrôlent leurs émotions, ne tombent pas dans la compassion, inhibent leur irritation ou leur colère comme leurs (rares) moments de satisfaction professionnelle, se soutiennent en cas de problème, cultivent une connivence ostensible au sein des collectifs sectorisés de travail... Ce travail est perçu comme en évolution négative : l'office des poursuites ne parvient plus à satisfaire la masse des sollicitations, et les dossiers non aboutis s'accumulent. Ils ressentent un sentiment croissant d'impuissance, et ont souvent la tentation de baisser les bras malgré une ferme volonté d'assumer la responsabilité de leurs décisions individuelles. Ceci s'accompagne d'une certaine nostalgie et d'interrogations quant au sens de ce métier.

8 Le travail des huissiers est principalement composé de trois configurations distinctes mais articulées : l'instruction solitaire des dossiers des débiteurs qui viennent sur leurs bureaux numériques, l'interaction au guichet avec les débiteurs se présentant à leur initiative ou sur convocation, les visites à domicile dans l'objectif de compléter la documentation des dossiers, de procéder à l'inventaire des biens saisissables, et/ou de saisir ces biens.

9 La configuration d'interaction au guichet seule sert à illustrer le propos de cet article. Sous pression temporelle, les huissiers établissent avec les débiteurs les modalités d'une coopération toujours singulière mais tenue par les rubriques ordonnées d'un document administratif : le formulaire F6. La coopération pour renseigner ce dossier se constitue entre doute mesuré et confiance prudente à l'égard des dires des débiteurs. Elle est aussi documentée par les connaissances préalables : des mémentos personnels, des informations stockées sur la plateforme numérique utilisée par tous, des savoirs glanés sur le terrain ou via des informateurs non officiels. Elle est ajustée au niveau de compréhension et de culture administrative des débiteurs, marquée par l'évolution des positions et attitudes en cours d'interrogatoire, et jalonnée par l'élaboration d'hypothèses transitoires.

10 Cette configuration se concrétise par le renseignement des rubriques du formulaire F6 et la documentation de leur décision de saisir ou non les débiteurs. Cela se fait par la co-construction avec eux d'une histoire dont ceux-ci sont les protagonistes centraux, et l'explicitation de la démarche administrative et juridique propre à chaque dossier. Les séquences d'activité qui la composent sont tenues par des dilemmes dont les plus saillants sont: réduire la durée de traitement des dossiers versus les traiter en profondeur, mettre raisonnablement et systématiquement en doute la parole des débiteurs versus leur allouer du crédit, appliquer ou suivre les procédures versus faire comprendre les procédures et les décisions, faire fonctionner un cadre administratif et légal versus s'alimenter aux histoires singulières des débiteurs.

\section{Filmage et analyse du travail des huissiers}

11 L'analyse du travail, dont les résultats sont résumés ci-dessus, suppose l'accord et la coopération des acteurs impliqués. Les huissiers, volontaires et intéressés à l'enquête sur leur pratique, étaient disponibles pour être observés et filmés dans des conditions qui n'entravaient pas la réalisation de leur travail. Les usagers ont accepté d'être observés et enregistrés en audio. 
Lors du filmage la caméra était disposée sur pied, de 3/4 arrière par rapport aux huissiers assis au guichet, et en légère plongée. Le plan captait en partie les deux écrans d'ordinateurs disposés sur leur bureau (Figure 1). En fonction des gestes des huissiers, la caméra focalisait plus ou moins sur des éléments lisibles sur les écrans par des zooms arrière / avant et des mouvements latéraux. Un microphone HF discret, disposé sur la table entre huissiers et débiteurs, et relié à la caméra, enregistrait les échanges verbaux.

Image 1

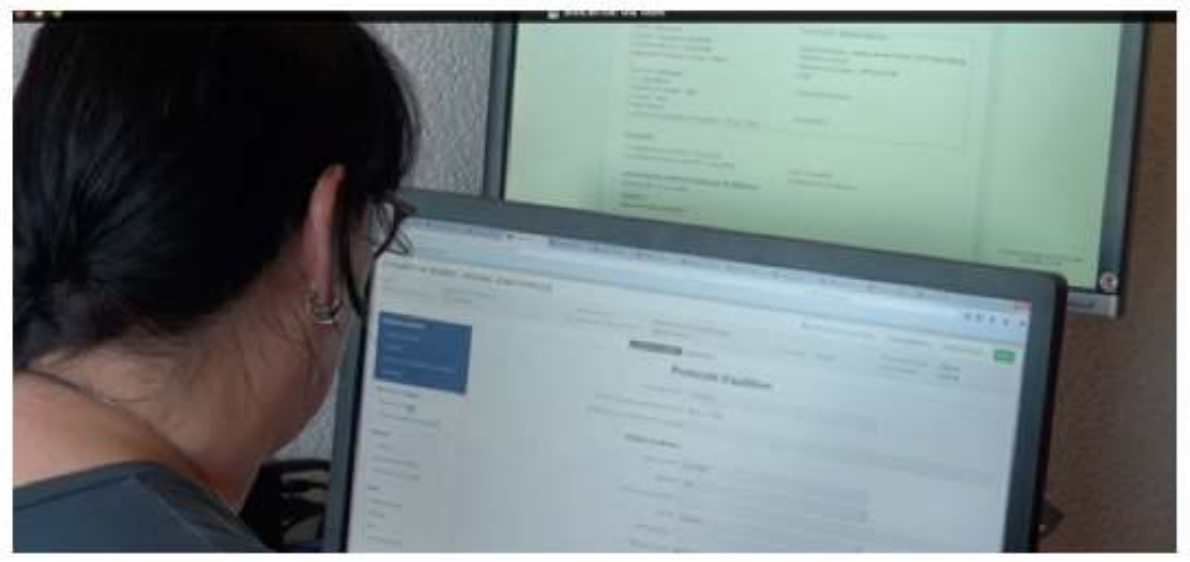

Exemple de plan pour le filmage de l'activité d'une huissière lors d'une interaction au guichet avec un débiteur (situé hors champ en avant et sur la droite de l'huissière).

13 Ces rushes enrichissent et complètent les observations directes, qui permettent des visionnements répétés, avec des arrêts sur image, des ralentis, des loupes et des retours en arrière. De plus, ils supportent les étapes successives de l'analyse de l'activité, en favorisant le consensus entre analystes lors du codage séparé des épisodes de travail. Ils sont enfin utilisés comme supports de recueils de données complémentaires relatives à l'expérience de travail des huissiers, ainsi qu'à ses contraintes et effets.

\section{Les données relatives à l'expérience de travail}

L'expérience est conceptualisée comme inhérente à l'activité humaine - et notamment l'activité de travail -. Toute pratique s'accompagne d'une présence à soi fluctuant au fil de son inscription dans le temps (Theureau 2006). Cette expérience est un vécu, tel que si une partie de leur activité échappe à la conscience des acteurs (elle est automatique ou inconsciente), une autre partie de cette activité est consciente ou susceptible de l'être. Ces acteurs ont la capacité d'exprimer/expliciter leur vécu lié à l'accomplissement pratique moyennant certaines conditions.

15 Cette capacité est exploitée afin de compléter les données d'observation par des données propres au vécu des acteurs lors de leur travail. A posteriori ceux-ci explicitent leur expérience lors d'une Activité 1 (passée), et la rende accessible à l'analyste au cours d'une Activité 2 (actuelle). Cela nécessite qu'ils soient alors en situation de remise en situation dynamique, c'est à dire engagés avec l'intention principale de présentifier ou revivre l'Activité 1 lors de l'Activité 2 (Theureau 2010), c'est à dire qu'un 
re-enactment $t^{1}$ se produise à partir du visionnement des traces vidéo de leur Activité 1. L'expression de cette expérience passée est une source d'information sur les significations de l'Activité 1 et sa dynamique intrinsèque pour les acteurs. Elle complète l'observation préalable réalisée par l'analyste. Des démarches ont été conçues pour favoriser l'accès à cette expérience passée et son expression : les auto-confrontations (Theureau 2010).

Dans le cadre de notre intervention, les professionnels étaient invités - et accompagnés en cela par deux analystes - à se remettre individuellement en situation dynamique lors de l'Activité 2, à se confronter aux rushes en se replongeant dans l'Activité 1 afin d'exprimer (ou rendre explicite) leur expérience au moment où s'était déroulé l'épisode visionné. Par ses consignes et relances, un des analystes s'efforçait de favoriser un dépliement et une amplification de l'expérience vécue passée (l'autre analyste étant derrière la caméra à filmer et observer silencieusement). Cette aide consistait en une incitation du professionnel à revivre l'épisode filmé et à éviter une attitude de jugement, explication ou généralisation de l'Activité 1 durant l'Activité 2. L'analyste insistait pour obtenir l'expression la plus précise et détaillée possible de l'expérience en cours d'Activité 1 . Pour sa part, il se référait à une appréhension empathique de l'Activité 1 , à son expérience d'analyste du travail en général, et à sa connaissance de ce travail-ci. Il était tenu par un cadre théorique précis, selon lequel cette expérience est constituée de six composantes correspondant approximativement à ce qu'on entend par intentions, attentes, savoirs, perceptions sensations et souvenirs, actions (symboliques ou pratiques), et généralisations (Theureau 2006).

17 L'analyste aidait l'expression (verbale et non verbale) de cette expérience par des arrêts sur image, des ralentis, des retours en arrière et des incitations relatives à l'attitude du professionnel. Ses relances étaient orientées vers l'expression de l'expérience à ce moment-là, en excluant les généralités (par exemple : «C'est ce que vous ressentez, là ? A cet instant-là... ? ; « Vous vous dîtes quelque-chose là, lorsqu'il vous répond... ?»). Elles étaient dépourvues de contenu (par exemple: «Et quand vous voyez cela, vous voyez quoi...? »), ou proposaient un questionnement ouvert (par exemple: «Et là, précisément, vous faites quoi...?»), et reprenaient la fin de l'énoncé précédant de l'acteur (par exemple: « ... Ça s'annonce mal dîtes-vous...? »). Enfin elles précisaient une demande orientée vers la connaissance de composantes de l'Activité 1 (par exemple: «En posant cette question que recherchez-vous?»,»Etait-ce la même recherche que pour votre question précédente?»).

L'expression de l'expérience passée est favorisée par le choix de cadrage et de prise de vue lors du tournage qui, outre le fait de renseigner sur ce que font les professionnels, les incitent à se focaliser sur leur propre activité à cet endroit-là et à ce moment-là, lors de l'auto-confrontation. Dans le cas des huissiers, les plans de l'interaction au guichet excluant les débiteurs relevaient de décisions relatives à l'objet de l'enquête (i.e. l'activité des huissiers pendant l'interrogatoire dans le but de renseigner le formulaire F6) et aux contraintes de confidentialité relatives aux usagers).

19 L'organisation du visionnement de l'Activité 1 concrétisait un emboîtement des Activités 1 et 2, et une mise en abîme : le film montrait l'huissier quasiment de dos en présentant sa nuque et l'arrière de sa tête lors de son Activité 1 et une partie de l'environnement de travail presque comme le ferait une caméra subjective. De plus, le positionnement de l'analyste et de l'huissier épaule contre épaule, face à l'écran et penchés sur lui, favorisaient la dynamique de re-enactment et d'immersion empathique, 
ainsi que de parler à l'épaule focalisé sur ce qui est sur l'écran, tout en dissuadant l'installation d'une discussion usuelle en face à face (Figure 2).

Les séances d'auto-confrontation étaient filmées, la caméra placée de dos, centrée sur l'écran présentant l'Activité 1 . Ceci permettait de faire coïncider précisément et $a$ posteriori l'expérience exprimée par les professionnels et les composantes observables de leur activité.

Image 2

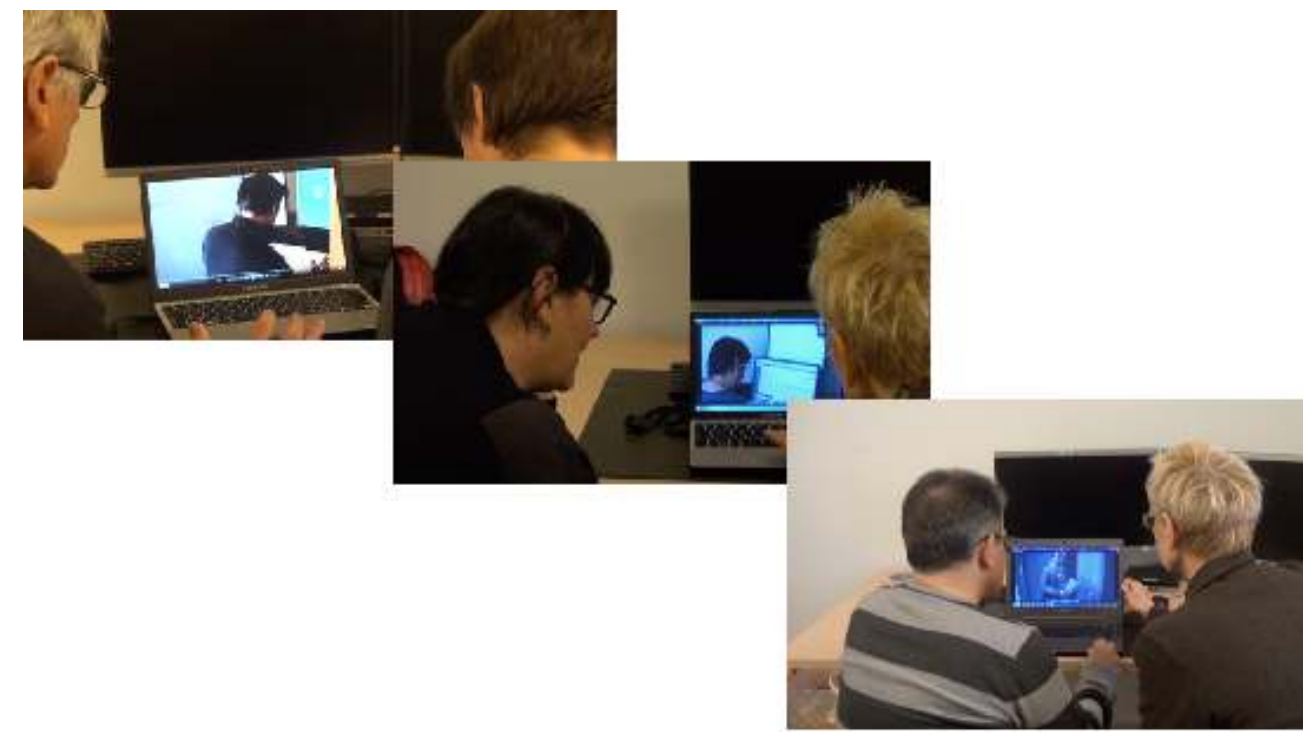

Exemples de configuration des séances d'auto-confrontation au cours desquelles les huissiers étaient aidés par l'analyste au re-enactment et à l'expression de leur expérience lors de l'Activité 1 .

\section{Les données relatives aux contraintes et effets sur l'activité}

Après l'auto-confrontation, un recueil complémentaire de données est réalisé à partir du visionnement des rushes, et animé par les deux analystes dont l'un avait conduit l'auto-confrontation. Ce recueil porte sur l'environnement, le corps phénoménal ${ }^{3}$ et la culture des acteurs. Il est orienté vers la description par eux des contraintes et des effets sur leur activité afin de compléter la description réalisée par l'analyste à partir de données externes ou objectives (Theureau 2006). Il est demandé aux acteurs de se détacher de leur expérience lors de l'Activité 1 tout en s'y référant, et de la (re)placer dans la dynamique historiquement, culturellement et spatialement située des contraintes qui pèsent sur elle, et de ses produits ou effets tangibles. Contraintes et effets de l'activité du professionnel sont conceptualisés comme ressortissant de son corps vécu et individué (ses dispositions, son histoire...), de sa culture en acte (ses ambitions, ses goûts et préférences, ses valeurs, sa signification de ce métier...) et de son environnement (sa tâche et son contexte socio-organisationnel, ses objectifs, les exigences managériales, les rapports hiérarchiques...) (Theureau 2006).

Cette situation comptait quatre pôles : le professionnel, l'écran, l'Analyste A, l'Analyste B. Les rushes provenant du tournage sur la place de travail et des auto-confrontations constituaient le point de centration du recueil de données et des échanges entre le professionnel et les deux analystes. Tout en concrétisant la référence à l'épisode visionné, le professionnel était incité à s'en détacher pour s'adresser aux analystes 
(Figure 3). L'Analyste A se tenait à ses côtés et éloigné de l'écran ; il interagissait avec le professionnel en face à face à propos de l'Activité 1. L'Analyste B qui se tenait en arrière et à côté de la caméra, renforçait par sa position et ses questions la prise de distance avec l'Activité 1 . Les aides et relances par les deux analystes concernaient les domaines suivants: les normes et habitudes qui particularisent l'activité en question et ses différences avec d'autres pratiques sociales, la dynamique de son émergence en lien avec ses contraintes, ses effets sur l'individu son environnement et sa culture, son partage avec d'autres professionnels dans le collectif de travail, et son évolution historique individuelle et collective. ${ }^{4}$

Image 3

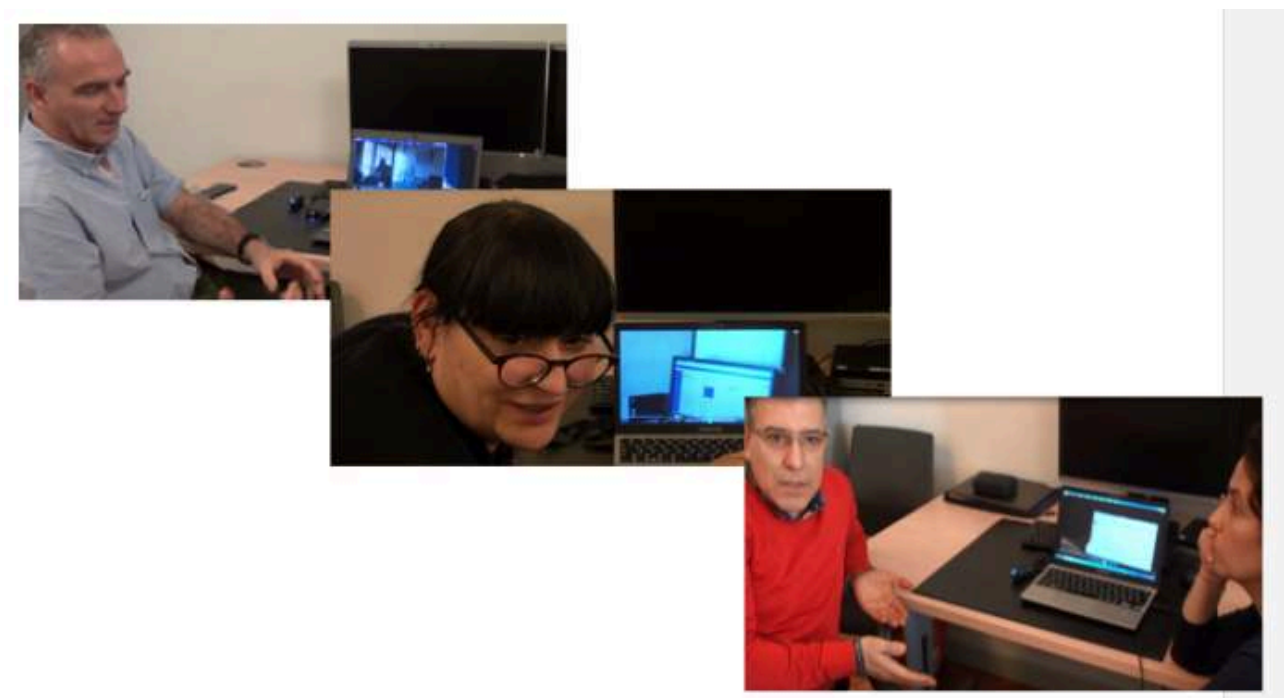

Lors du visionnement de leur Activité 1, les huissiers étaient incités à décrire la dynamique «contraintes extrinsèques - effets extrinsèques » de leur travail et son évolution. La configuration adoptée les invitait à se tourner et se détourner momentanément des images filmées (dont le défilement était interrompu).

Cette séance relative aux contraintes et effets extrinsèques de l'activité était filmée en plan moyen, caméra sur pied, un analyste derrière - ou proche de - la caméra.

\section{Images filmées, conception d'objets temporels documentaires et situations de formation}

L'ensemble du corpus filmique est constitué de traces d'activité différentes. L'analyste réalise une synthèse de cette hétérogénéité en allouant un primat au point de vue des acteurs sur leur activité. La démarche d'analyse procède en trois étapes: la reconstitution des cours d'activité de chaque acteur dans chaque situation ou épisode de travail, le repérage de ressemblances ou singularités entre les sous-corpus (plusieurs professionnels et plusieurs situations), et enfin l'identification et reconstitution d'épisodes-types conceptualisés.

Dans leur activité quotidienne, les acteurs tendent à généraliser leurs expériences singulières et à leur conférer un potentiel expansif susceptible de recouvrir des situations autres mais ayant un air de famille (Rosch 1973; Theureau 2006). Chaque expérience est potentiellement à l'origine de la constitution d'une typicalité, c'est à 
dire de nouveaux types ou de la transformation de types anciens; et ces types constituent les ressources à disposition des acteurs et des collectifs, à partir desquelles ils signifient les situations nouvelles. Ils remplissent ainsi une double fonction conservatrice et novatrice au plan cognitif en signifiant la nouveauté à partir du déjà connu et déjà vécu, sans la réduire à ce déjà-là. Au plan social ils définissent dans l'action les degrés de légitimité et validité des pratiques (Lave 2012). Dans cette dynamique, le repérage de relations de ressemblance (d'air de famille) grâce aux métaphores et aux significations imagées est essentielle (Fisette 2008; Salini et Durand 2016).

L'analyse du travail réel des acteurs permet notamment le repérage de ses composantes typiques, c'est à dire d'occurrences identifiées et évaluées comme les meilleures représentantes de l'échantillon d'épisodes étudiés et, par extension, du travail en question. Si l'analyste est pertinent, la typicité ainsi repérée est analogue à celle construite par les acteurs dans leur activité quotidienne. Le caractère de typicité renvoie à cinq aspects au moins: descriptif (l'occurrence type concentre le plus d'attributs de l'activité observée au sein de l'échantillon des acteurs et des situations étudiés), statistique (l'occurrence-type est la plus fréquemment observée dans l'échantillon enquêté), génératif (le type présente une propension à s'actualiser de façon privilégiée lorsque des conditions ayant un air de famille avec celles observées sont reproduites), significatif (les acteurs expriment un sentiment de typicité lorsqu'ils sont interrogés à ce propos), et expansif (l'analyse révèle des ajustements, des migrations et des diversifications des types dans le temps et selon les environnements) (Durand 2014 ; Poizat et al. 2016).

Une fois ce caractère de typicité validé par les professionnels, ces épisodes et situations typiques alimentent une modélisation du travail (en termes de dilemmes et situations ou activités-types), et constituent le cadre de référence pour la conception des formations.

\section{La conception de films de formation sur le travail}

Les rushes constituent le corpus à partir duquel sont conçus des films pour la formation. Ceux-ci ont des propriétés fonctionnelles procédant de l'analyse et de la modélisation du travail, et de nos hypothèses relatives à l'activité humaine et ses transformations (Durand 2017 ; Poizat et al. 2016).

Les films sont des objets temporels (Stiegler 2010) : ils changent et s'écoulent dans le temps. Par un processus de synchronisation avec le flux de l'image, ces transformations s'accompagnent d'une captation du flux de conscience et d'expérience du spectateur/ visionneur, que cet auteur caractérise comme un pouvoir d'envoûtement des objets qui passent. Ce pouvoir tient à ce qu'ils disparaissent à mesure qu'ils apparaissent: la condition de leur apparition à la conscience des acteurs est leur disparition.

Utilisé en formation, ce pouvoir de captation des objets temporels suppose et supporte des phénomènes mimétiques et empathiques chez les participants: le visionnement d'un film déclenche chez l'Huissier B visionneur, un engagement de type «l'autre comme soi-même » au spectacle du travail de l'Huissier A. Il est à l'origine d'une expérience caractérisable par une double négation : pour l'Huissier B l'activité observée n'est pas la sienne, mais elle n'est pas "pas la sienne» (Horcik et Durand 2015). Saillante dans le jeu, l'art, la fiction, et convoquée dans certains dispositifs de 
formation, cette expérience particulière caractérise un engagement à la situation qui, même s'il est pragmatique et orienté vers la production et le travail, constitue simultanément un lâcher-prise propice à des transformations majorantes de l'activité.

31 Les films de formation sont des promesses de captation de l'expérience des participants aux formations. Cela suppose qu'ils aient une durée correspondant à un empan d'activité conscientisable et re-saisissable en une nouvelle expérience globale (que nous estimons à 7-8 minutes). Par ailleurs leur contenu doit être significatif pour les participants, et ressenti comme typique du travail en question. Nous résumons ces propriétés par l'idée que le contenu de ces films doit être appropriable.

Les rushes sont montés avec l'intention de mettre en récit des épisodes typiques de travail. Les films sont des reconstructions qui imagent un thème ou une composante critique du travail sous forme de micro-histoires selon un schéma narratif classique: une situation initiale qui lance le récit, un élément déclencheur ou accrocheur qui perturbe la situation initiale, un enchaînement d'actions ou d'interactions qui thématisent le récit et lui confèrent sa spécificité, un dénouement portant souvent la décision prise par l'huissier et conduisant à la situation finale. Produire ce schéma narratif suppose d'avoir opéré des sélections et des découpages dans les rushes, modifié la chronologie de l'épisode et supprimé certaines de ses parties, introduit des répétitions, mis en saillance tel aspect, etc.

Cette démarche de mise en récit relève de trois hypothèses à dimension anthropologique : celle relative à la construction et compréhension du monde et de soimême selon des formes narratives (Bruner 2003), celle d'une propension à signifier le réel et les flux événementiels, ainsi qu'à piloter ses propres actions en les insérant dans des cas, ce qu'on peut synthétiser par la formule : penser et agir par cas (i.e. Passeron et Revel 2005), et celle portant sur la prégnance dans les récits structurés et culturellement validés, de schémas narratifs ou actantiels généraux et généralisateurs (Greimas 1966; Propp 1970). Au plan technique, cette démarche est à rapprocher du courant du digital storytelling qui propose des productions non exclusivement langagières de ces récits par le recours à des outils numériques ou vidéo dans des intentions éducatives ou thérapeutiques (Lambert 2006 ; Rossiter et Garcia 2010).

Le montage ne répond pas à une intention évidente de cinéma du réel. Ces films sont doublement documentaires au sens où ils rendent compte d'une réalité et sont conformes à la modélisation préalable du travail, et où ils documentent un usage particulier en formation. Le montage puise dans le corpus des rushes provenant des diverses données recueillies: filmage des pratiques, expression de l'expérience, des contraintes et effets extrinsèques, etc. Il répond à la recherche d'effets précis et explicites. Ainsi par exemple, afin de rendre compte d'un épisode au cours duquel un huissier soumet le débiteur à un feu roulant de questions factuelles, les réponses de l'usager sont supprimées au montage pour ne conserver que les questions, avec une coupe au plus serré au début et la fin de chaque question, et les questions éparses dans l'épisode réel sont rassemblées et montées avec des transitions fondues-enchaînées très brèves qui procurent une impression de bouger sur place. Ce montage accentue la signification du travail au guichet comme une compilation active et contrainte d'informations, de pression exercée sur le débiteur et de rigueur dans la succession des questions (qui correspondent aux catégories du formulaire F6).

Enfin, dans certains cas, des insertions de textes s'affichent brièvement en bas d'écran. Ces énoncés qualifient la séquence en cours (Figure 4) et signifient de façon synthétique 
la portion d'activité visionnée, en condensant notamment les dimensions saillantes de l'expérience des huissiers (intention, action, émotion, sensation, perception, attente...). Ils sont présentés sous une forme laconique : la plupart du temps par un verbe d'action à l'infinitif et un complément d'objet direct ou indirect (par exemple: Masquer ses émotions ; Enoncer le problème ; Interrompre poliment...). Ils sont destinés à ouvrir sur une généralisation et une élaboration symbolique de ce qui est montré.

Image 4

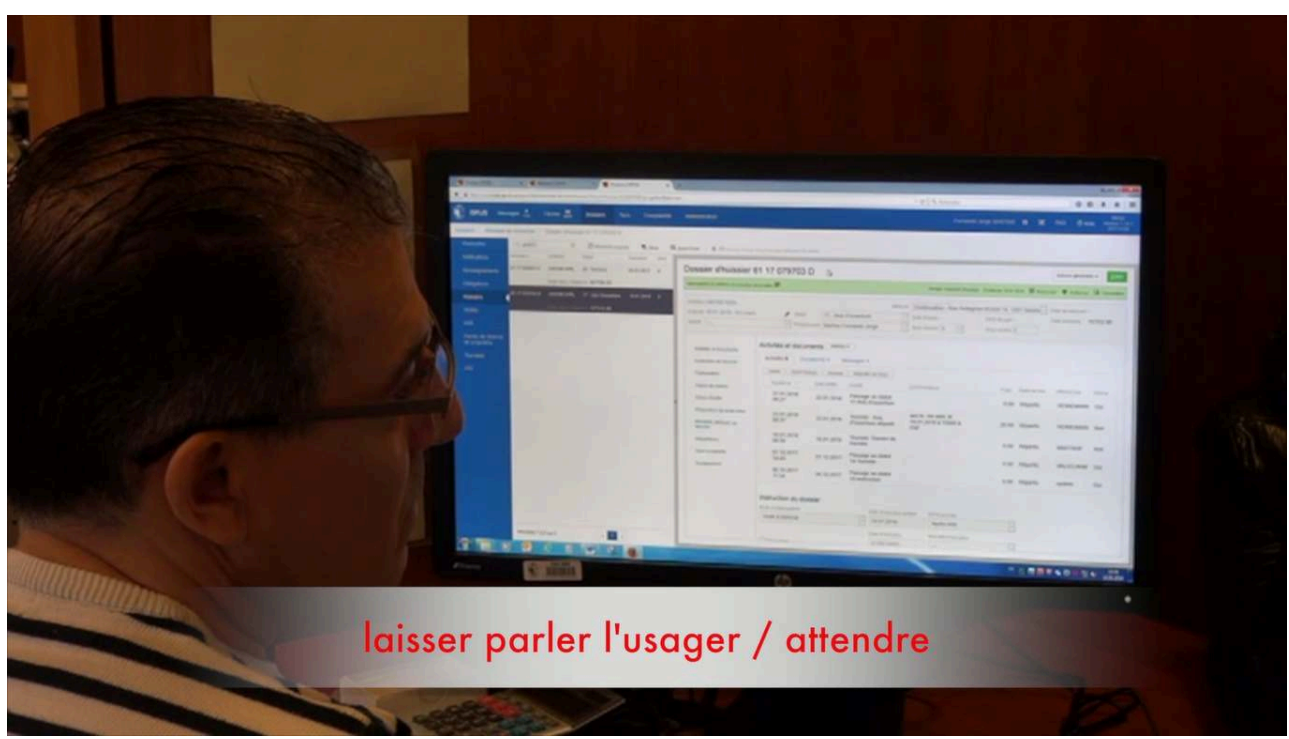

Extrait d'un film présentant un « type d'action » et la rendant explicite pour les participants aux formations.

\section{La formation instrumentée par les images filmées}

Les situations de formation visent des transformations de l'activité des participants (Durand et Poizat 2015). Ceci suppose de leur proposer des situations qui soient perturbatrices ou disruptives au sens où elles marquent une rupture dans la continuité de leur activité et s'accompagnent d'une instabilité qui s'installe ou non. Elles sont prometteuses d'une réorganisation de cette activité retrouvant une stabilité antérieure ou trouvant une stabilité nouvelle. Les situations sont plus ou moins appropriables selon les participants, mais une promesse d'appropriabilité des films réside, selon nos hypothèses, dans le fait d'exposer des contenus typiques du travail en question. Elles sont formatrices lorsque la perturbation déclenche une multi-stabilité caractérisée par la coexistence de différents possibles susceptibles de s'actualiser, ou une métastabilité c'est à dire un état instable et non déterministe, promesse d'une possible rééquilibration majorante (Simondon 2005).

\section{Mimétisme et re-enactment}

Les films offrent aux participants des possibilités d'engagement mimétique et de reenactment, conçus comme des vecteurs d'appropriation / expropriation des composantes typiques du travail. L'économie de cette démarche de formation repose sur deux orientations qui tiennent à ce que les films ne sont pas des spectacles à regarder mais des expériences à vivre, et à ce que visionner ces films conçus à dessein 
est susceptible de déclencher une activité significativement plus riche et complexe que la simple contemplation d'un enregistrement vidéo du travail.

Le visionnement de son propre travail mis en récit filmique, est le support d'un engagement mimétique et re-enactif, souvent empreint d'émotions (Leblanc et Sève 2012). Ce visionnement ouvre sur un début d'expropriation de son activité, celle-ci devenant un contenu ou un objet indépendant de l'acteur. Ceci la rend partageable avec d'autres acteurs du fait de sa présentation sous forme d'un récit construit en allouant un primat à l'intrinsèque (ce que l'on peut caractériser par la formule «son propre travail comme celui d'un autre »). Cette expropriation - qui est détaillée plus loin - est conçue comme fournissant une amorce de démarche de réflexivité augmentée et un contenu (l'activité de travail) pour cette démarche.

Le visionnement de l'activité d'un autre professionnel est aussi le support d'un engagement mimétique et re-enactif, parce que les épisodes visionnés sont typiques du travail, c'est à dire sources d'un partage de signification d'un vécu au moins partiellement commun. Si le récit filmé est pertinent il présente cette typicité qui appelle des jugements de ressemblance, d'accord ou désaccord sur un registre corporel et symbolique de perturbation ou non avec ce qui est visionné. Cet engagement mimétique / re-enacté est favorable à des transformations majorantes parce qu'il est propice à l'émergence de significations basées sur l'établissement d'analogies non logiques ou non conventionnelles, comme la perception d'un d'air de famille. Par un jeu de superposition d'expériences / activités qui sont convoquées, supportées, amplifiées et orientées, les récits filmiques favorisent la superposition des activités passées et actuelles, d'autrui et de soi-même. En cela ils sont des vecteurs de réflexivité au double sens d'un réfléchissement mimétique et d'une réflexion relative à l'identification et la validation de types d'action anciennes ou nouvelles.

\section{Appropriation et expropriation}

Le visionnement accompagné des films du travail est potentiellement à l'origine d'un dynamisme à double sens : une appropriation phénoménale, corporelle et symbolique des composantes de l'activité typique du métier objet de la formation, et une expropriation phénoménale, corporelle et symbolique, au sens où l'activité/expérience des professionnels visionnés leur échappe et constitue une altérité susceptible d'enrichir la culture de chaque autrui, et la culture commune du collectif de travail.

41 La dynamique d'appropriation est l'ensemble des transformations intégrées par lesquelles les individus, les situations et les cultures propres sont mobilisés et modifiés en fonction d'engagements praxiques répétés et / ou variés (Poizat 2014; Poizat et al. 2016). Ainsi, notre intervention étendue sur plusieurs mois a permis de repérer des transformations de l'activité des huissiers au guichet. Elles concernent notamment l'usage du nouvel environnement numérique. Aujourd'hui, les huissiers identifient sur l'écran et dans l'action, des indices qu'ils ne percevaient pas un an plus tôt; ils appréhendent globalement et quasi immédiatement des pages du formulaire F6 en lien avec une pondération des zones de l'écran explorées liée à la répartition des informations sur tout l'écran, et au caractère plus ou moins crucial pour leurs décisions. Au guichet, ils ne perçoivent plus l'outil numérique comme un obstacle ou un double écran physique entre eux et l'usager, mais comme une composante intégrée dans une expérience globale d'interaction. Les ordinateurs disposés sur le guichet font corps avec eux en situation, en matérialisant et portant leurs intentions, leurs actions 
et leurs façons de signifier le dossier en cours. Alors que jusqu'à huit pages du programme peuvent être ouvertes simultanément, l'une masquant les autres et étant seule visible, le passage d'une page à l'autre (et d'un écran à l'autre) sont devenus plus rapides. Une expérience continue associée à ces mouvements (décrits par un huissier comme proche du surf) s'est substituée d'une part à celle décrite un an plus tôt, de se perdre en tâtonnant dans l'empilement des pages, et d'autre part d'errer de l'une à une autre. Le masquage des pages pose moins de problème de mémorisation de leur contenu qui est maintenant ressenti comme à disposition pour agir. L'environnement s'est reconfiguré en fonction de l'engagement praxique de ces professionnels, et d'une organisation de l'activité de renseignement du formulaire F6 à partir d'un classement pragmatique des cas, selon des critères qui ne renvoient pas à des catégories juridiques, sociales ou informatiques mais à des gradients de praticabilité. Dougherty et Keller (1982) ont désigné ce processus de catégorisation pratique par le néologisme difficile à rendre en français de taskonomy (et non taxonomy) c'est à dire d'une classification en acte des objets et situations qui sont utiles pour l'action et ne répondent pas aux critères d'une logique aristotélicienne. ${ }^{5}$

Si les expériences mimétiques et de re-enactment déjà décrites sont présentes, les manifestations de ce processus d'appropriation sont aussi observables lorsque les situations dans lesquelles sont engagés les acteurs, sont des situations de visionnement et non d'effectuation du travail. De sorte que, à partir de son expérience du travail, l'acteur est susceptible d'avoir accès au vécu d'autrui, par une relation empathique incarnée. Il peut ainsi se l'approprier. En raison de l'intensité de la captation mimétique et re-enactive décrite plus haut, il se produit donc un phénomène d'extériorisation et d'aliénation, c'est à dire un devenir autre - qui n'est pas nécessairement chargé de valeurs négatives. Cette expropriation signe un partage désindividuant de la pratique, et appelle spontanément des débats et controverses dès que des pratiques analogues mais différentes sont visionnées. Cette interactivité empathique est une prémisse à la co-constitution d'acteurs agissants et d'un collectif de et au travail, notamment grâce à des appropriations mutuelles par les différents acteurs impliqués.

De façon concomitante, se manifeste donc un processus d'expropriation-appropriation simultanément individuel-collectif. Celui-ci tient à ce que l'activité visionnée grâce au film du travail, est aliénée, c'est à dire qu'elle devient potentiellement un objet extérieur à l'acteur filmé, et que cet objet devient potentiellement partagé par le collectif de formation et de travail, par une appropriation mutuelle ou collective (Lave et McDermott 2002). Ce processus présente une forte connotation corporelle, au sens où Merleau Ponty (1992 a) parle de chair du monde partageable. Grâce au film, l'activité et l'expérience qui étaient propres à un individu sont potentiellement projetées, au plan phénoménal, dans les autres individus ou dans leur monde culturel partagé. En d'autres termes, cette expropriation et ce partage font simultanément émerger une communauté centrée sur l'activité de travail. Ceci correspond aux puissants phénomènes de communauté de pratique étudiés par Lave (2012). Par exemple, il est impossible à un expert d'un métier d'observer un professionnel réalisant le même travail que lui, sans participer à son activité, et ce n'est qu'au prix d'un effort d'inhibition qu'il peut rester passivement à observer en totale neutralité cette activité comme un spectacle. Le visionnement des films de travail favorise cette captation de l'activité du professionnel visionnant celle d'un autre, dont les limites phénoménales sont poreuses, au point que l'on peut dire que cette communauté de la pratique une fois installée ouvre sur la constitution de communautés de praticiens. Sans cette 
participation, les collectifs de travail ne sont que des groupes d'individus engagés dans des tâches analogues ou complémentaires.

Dans l'expérience de travail et de visionnement du travail, il y a «toujours déjà » la place pour de l'activité d'autre que soi. L'acteur qui visionne cela est affecté par l'activité d'autrui mais il n'est pas à la place d'autrui ; une relation s'instaure qui n'est pas une fusion, mais une réciprocité qui maintient la différence. Lors des visionnements, il ne s'agit pas seulement de contempler l'activité de travail d'autrui comme analogue à la sienne: ce qui semble prometteur de développement et d'apprentissage est la subsistance d'un léger et mystérieux décalage porteur d'apparentement et d'opposition (et non pas d'identifications) (Merleau-Ponty $1992 \mathrm{~b}$, p.186). Pour paraphraser cet auteur on pourrait écrire que les films du travail opérationnalisent dans une visée de formation l'idée que pour un acteur «l'activité des autres hante sa propre activité ».

\section{Conclusion}

Les situations de formation centrée sur le visionnement de films du travail conçus comme cela est décrit ci-dessus, proposent de transcender la simple observation ou la perception lors du visionnement d'un film, pour une appréhension des fondements implicites et culturels de l'activité professionnelle (Suhr et Willerslev 2012) : l'enjeu et de passer du visionnage du film à l'appréhension de l'activité de travail. En raison de leur contenu, du montage et des conditions de leur visionnement, ces films véhiculent simultanément une propriété (c'est de l'activité propre), une altérité (ce qui dépasse l'activité propre) et une collectivité (ce qui est partagé et qui gomme les propriétés) qui sont appréhendables par les professionnels.

Utiliser les films pour révéler ces aspects invisibles du travail implique le maintien d'une tension entre une adhésion à la réalité de ce travail, et sa perturbation. Les réaliser suppose de surmonter une tendance de formateur et d'analyste du travail à produire des dispositifs filmiques réalistes copiant fidèlement la réalité. Ici le montage notamment, devient manipulateur et perturbateur de cet asservissement littéral à l'activité observable, afin d'évoquer l'invisible partageable de l'activité. Ce montage perturbateur fait que ces films documentent le travail sans être stricto sensu des documentaires (pas plus d'ailleurs que de pures fictions): leur fonction n'est pas d'informer sur ce travail, mais d'inciter à s'y plonger, et à conjoindre l'expérience de l'acteur filmé avec celle du visionneur. Leur visionnement permet la perception de l'activité de travail, et non pas seulement sa contemplation spectaculaire.

Ces films sont des moyens de conjuguer les dynamiques appropriatives des composantes du métier initialement étrangères aux novices, et expropriatives assurant la projection vers autrui et en des collectifs, des composantes de l'expertise de chaque professionnel, tout en les mettant en débat. Cette perspective centrée sur le filmage du travail et le travail du filmage, implique une re-définition large et intégrative des pratiques de formation professionnelle. Elle suppose aussi un rapport complexe de ces films du travail à visée formative, à la réalité du travail: ni fictions pures, ni documentations réalistes, ils proposent des interprétations orientées et construites des pratiques laborieuses à partir de l'analyse de l'activité in situ des professionnels. 


\section{BIBLIOGRAPHIE}

Bruner J. (2003), « The narrative construct of reality », Critical Inquiry, n 18, p. 1-21.

Collingwood, R.G. (1946), Idea of History, Oxford, Clarendon Press.

Dougherty, J. et Keller, C.M. (1982), « Taskonomy : A practical approach to knowledge structures », American Ethnologist, n 94, p. 763-774.

Durand M. (2008), « Un programme de recherche technologique en formation des adultes », Education et Didactique, $n^{\circ} 2$, p. 69-93.

Durand M. (2014), « Activité humaine, pratiques sociales et éducation des adultes », J. Friedrich et J. Pita (dir.) Un dialogue entre concepts et réalité en formation des adultes. Dijon : Editions Raison et Passions, p. 15-39.

Durand M. (2017), « L'activité en transformation », Barbier J-M. et Durand M. (dir.), Encyclopédie d'analyse des activités, Paris, PUF, p. 33-55.

Durand M. et Poizat G. (2015), « An activity-centred approach of work analysis and the design of vocational situations », Filliettaz L. et Billett S. (dir.), Francophones perspectives of learning through work, Heidelberg, Springer, p. 221-240.

Fisette J. (2008), « La rencontre de la sémiotique et de l'esthétique chez Peirce », Recherches Sémiotiques, $\mathrm{n}^{\circ} 28, \mathrm{p} .31-56$.

Greimas, A. (1966). Sémantique structurale : recherche de méthode, Paris, Larousse.

Horcik Z. et Durand, M. (2015), « L'expérience mimétique dans l'apprentissage adulte : le cas des formations par simulation », Revue Suisse des Sciences de l'Education, n 37, p. 167-186.

Lambert J. (2006), Digital storytelling, Berkeley, Digital Diner Press.

Lave J. (2012), «Changing practice », Mind, Culture and Activity, n 19, p. 156-171.

Lave J. et McDermott R. (2002), « Estranged labor learning », Outlines, n 1, p. 19-48.

Leblanc S. et Sève C. (2012), « Vidéoformation et construction de l'expérience professionnelle », Recherche et Formation, $\mathrm{n}^{\circ}$ 70, p. 47-60.

Merleau-Ponty M. (1945), Phénoménologie de la perception, Paris, Gallimard.

Merleau-Ponty M. (1992 a), La prose du monde, Paris, Gallimard.

Merleau-Ponty M. (1992 b), L'œil et l'esprit, Paris, Gallimard.

Passeron J-C. et Revel J-F. (dir.) (2005), Penser par cas, Paris, Edition EHESS.

Poizat, G. (2014), « Le concept d'appropriation en formation des adultes », Friedrich J. et Pita J. (dir.), Un dialogue entre concepts et réalité, Dijon, Raison \& Passions, p. 40-68.

Poizat G., Durand M. et Theureau J. (2016), « Challenges of activity analysis oriented towards professional training ", Le Travail Humain, ${ }^{\circ}$ 79, p. 233-258.

Propp, V. (1970), Morphologie du conte, Paris, Seuil.

Rosch E.H. (1973), « Natural categories », Cognitive Psychology, n 4, p. 328-350.

Rossiter M. et Garcia P.A. (2010), « Digital storytelling : A new player on the narrative field », New Directions for Adult and Continuing Education, $\mathrm{n}^{\circ}$ 126, p. 37-48. 
Salini D. et Durand M. (2016), « Evénement dramatique et éducation événementielle », Assal J-P., Durand M. et Horn O. (dir.), Le Théâtre du Vécu. Dijon, Raison et Passions, p. 265-276.

Simondon G. (2005), L'individuation à la lumière des notions de forme et d'information, Grenoble, Millon.

Stiegler B. (2010), La technique et le temps. Le temps du cinéma et la question du mal-être, Paris, Fayard.

Suhr C. et Willerslev R. (2012), « Can film show the invisible? The work of montage in ethnographic filmmaking », Current Anthropology, n 53, p. 282-301.

Theureau J. (2006), Le cours d'action : méthode développée, Toulouse, Octarès.

Theureau J. (2010), « Les entretiens d'autoconfrontation et de remise en situation par les traces matérielles et le programme de recherche " cours d'action », Revue d'Anthropologie des Connaissances, $\mathrm{n}^{\circ} 4$, p. 287-322.

Theureau J. (2016), Enaction \& expérience, Toulouse, Octarès.

\section{NOTES}

1. Nous nous inspirons ici de la notion de re-enactment proposée par Collingwood (1946) dans ses travaux d'épistémologie de l'histoire.

2. Cette formulation est inspirée des codes scéniques de l'opéra au tournant des $19^{\text {ème }}$ et $20^{\text {ème }}$ siècle lorsque les acteurs-chanteurs se tenaient côte à côte face au public et sans se regarder lors des duos, tout en s'adressant l'un à l'autre.

3. Parler de corps phénoménal revient à insister sur l'idée que le corps d'un acteur n'est pas un objet pour une conscience séparée de lui mais la condition de cette conscience. C'est par le corps qu'il perçoit, configure et expérimente son monde propre et signifiant, de sorte que son activité est l'expression de son engagement incarné au monde (Merleau-Ponty 1945).

4. Il est difficile, par manque de place, de justifier ici l'ensemble des composantes et objectifs de cette démarche, ainsi que de la mettre en discussion avec d'autres méthodes. Nous renvoyons pour cela à des synthèses et mises en débat déjà publiées (Poizat et al. 2016; Theureau 2006, 2010).

5. Le nouveau logiciel était à l'origine d'une souffrance chez les huissiers qui avait motivé la demande d'intervention adressée à notre équipe. Cette appropriation sur le tas du logiciel au cours d'une année d'utilisation, nous paraît indiquer que, contrairement à l'opinion de la hiérarchie de l'office, la souffrance et les inquiétudes des huissiers était davantage liées à un affaissement de la signification de leur métier (la plainte à ce propos se fait d'ailleurs plus nette et précise un an plus tard) qu'à un manque momentané de maîtrise informatique.

\section{RÉSUMÉS}

Cet article présente des principes de conception de films portant sur le travail à des fins de formation professionnelle, et les illustre par un cas pratique issu d'un partenariat avec les huissiers d'un Office cantonal suisse. Ces principes reposent sur une analyse préalable de leur activité, de ses contraintes et de ses effets à partir de l'observation et du filmage du travail. Le 
montage secondaire des films s'éloigne de la présentation fidèle du travail pour en restituer les éléments typiques implicites, révélés par l'analyse préalable. Montés sous forme de récits, ils sont censés perturber les visionneurs en provoquant chez eux un engagement mimétique par rapport aux scènes présentées et un re-enactement lors du visionnage, c'est à dire une expérience proche du vécu de travail. Ce visionnage est aussi censé déclencher des dynamiques d'appropriation des composantes typiques du travail, et de leur expropriation et partage avec d'autres professionnels, favorisant la constitution d'une communauté de pratique.

This paper is about film editing for documentation of work practices and training purposes. It illustrates these principles by a practical case: the working activity of bailiffs in a Swiss cantonal office. Training situations designing is based on a prior analysis of bailiffs' activity, its constraints and its effects, by the mean of observation and video recording of work practices. During films editing, we move away from the faithful presentation of work practice, to account of its typical implicit elements, revealed by the preliminary analysis. The films are built like stories. They are supposed to disturb the viewers by provoking a mimetic engagement at them when viewing the scenes, and a re-enactement - i.e. an experience close to the former working one -. Viewings are also supposed to trigger appropriation of the typical components of activity by the bailiffs, and an expropriation and sharing of them with other professionals, promoting the construction of a community of practice.

\section{INDEX}

Mots-clés : analyse de l'activité, appropriation, communauté de pratique, expérience, expropriation, mimétisme, montage, re-enactment

Keywords : activity analysis, appropriation, community of practice, experience, expropriation, mimesis, movie editing, re-enactment

\section{AUTEURS}

\section{MARC DURAND}

Marc Durand est professeur honoraire et membre associé de l'Unité CRAFT (Conception, Recherche, Activité, Formation, Travail) de l'Université de Genève. Ses travaux portent sur l'analyse de l'activité humaine dans différents contextes et conduits selon une perspective enactive et phénoménologique. Ces recherches sont finalisées par la documentation et la conception de formations professionnelles et d'éducation tout au long de la vie. Il a récemment coordonné avec J-M. Barbier, l'« Encyclopédie d'analyse des activités » en 2017 aux PUF ; et « Représenter - Transformer » en 2018 chez L'Harmattan.

\section{ANNIE GOUDEAUX}

Annie Goudeaux est chargée d'enseignement au sein de la Section des Sciences de l'éducation de l'Université de Genève. Membre de l'équipe CRAFT (Conception, Recherche, Activité, Formation, Travail) de L'université de Genève. Ses travaux et intérêts de recherche portent sur le rôle de la technique dans le développement de l'activité humaine et ses conséquences en formation. Elle assure depuis deux ans la coresponsabilité d'un cours de Master en formation des adultes sur le vidéo documentaire en éducation et formation des adultes. Elle mène régulièrement des interventions dans les organisations à l'aide de médias vidéos sur le travail. 


\section{GERMAIN POIZAT}

Germain Poizat est professeur à l'Université de Genève, dans le domaine de la Formation des adultes et de l'analyse du travail. Il dirige l'Unité CRAFT (Conception, Recherche, Activité, Formation, Travail) et conduit des recherches dans différents contextes sociaux et organisationnels (e.g., industrie nucléaire, exercices de crise, services publiques, industrie de luxe...). Ses recherches s'inscrivent principalement dans le champ de l'analyse du travail et de la formation (tout en étant ouvertes sur d'autres pratiques sociales). Elles sont centrées sur l'activité et ses transformations en situations de travail et/ou de formation, et se caractérisent par le fait d'être menées sous les hypothèses de l'enaction et de l'expérience. Il a publié récemment l'article « Analyse de l'activité humaine et éducation des adultes : faits et valeurs dans un programme de recherche finalisée » coécrit avec Marc Durand dans la Revue française de pédagogie en 2015, ou encore l'article « Analyse du travail pour la formation : essai sur quatre problèmes méthodologiques et le recours à des synopsis d'activité » coécrit avec Simon Flandin et Luc Ria dans la Revue TransFormations en 2019. Il a également coordonné avec Mireille Bétrancourt l'ouvrage «Technologies numériques, e-formation, et éducation des adultes » en 2018 chez De Boeck.

\section{JUANA SARMIENTO JARAMILLO}

Juana Sarmiento est consultante indépendante, doctorante à l'Université de Genève dans le domaine de la formation des adultes et membre de l'équipe CRAFT (Conception, Recherche, Activité, Formation, Travail) de l'université de Genève. Ses travaux de recherche et d'intervention dans le cadre d'organisations publiques ou privées ainsi que dirigées à un public en conditions de vulnérabilité, s'intéressent à la conception et mise en œuvre de dispositifs de formation visant des processus d'apprentissage / développement / empowerment. 